\title{
Color Doppler ultrasound and contrast-enhanced ultrasound in the diagnosis of lacrimal apparatus tumors
}

\author{
YING-XIAN LIU ${ }^{1}$, YAN LIU ${ }^{1}$, JIN-MEI XU $^{1}$, QIN CHEN $^{2}$ and WEN XIONG ${ }^{2}$ \\ ${ }^{1}$ Department of Ultrasound Diagnosis, China Meitan General Hospital, Beijing 100028; \\ ${ }^{2}$ Department of Ultrasound Diagnosis, Sichuan Academy of Medical Sciences and \\ Sichuan Provincial People's Hospital, Chengdu, Sichuan 610072, P.R. China
}

Received December 5, 2017; Accepted May 11, 2018

DOI: $10.3892 / 01.2018 .8879$

\begin{abstract}
Color Doppler ultrasound and contrast-enhanced ultrasound (CEUS) in the diagnosis of lacrimal apparatus tumors were investigated. In total, 48 patients undergoing preoperative two-dimensional and color Doppler ultrasound and CEUS examinations were included in this study. Conventional ultrasound and CEUS characteristics of 48 patients pathologically and clinically diagnosed with lacrimal apparatus tumors were retrospectively analyzed. Results of conventional ultrasound of 29 cases with pleomorphic adenoma of lacrimal gland showed moderate-hypoechogenic solid masses in lacrimal gland; CEUS displayed two enhancement modes: High, fast-developed slow-extinct and overall uniform enhancement (20/29, 68.97\%) and high, fast-developed slow-extinct, centripetal, uniform or non-uniform enhancement (9/29, 31.03\%); after enhancement, the mass edge was clear without changes in size. Results of conventional ultrasound of 6 cases with adenoid cystic carcinoma of lacrimal gland showed hypoechogenic solid masses with unclear edge, irregular form, non-uniform echo, and abundant blood flow signals; the CEUS displayed high, fast-developed fast-extinct and overall uniform enhancement; after enhancement, mass edge was unclear and masses were larger than that in two-dimensional ultrasound. Results of conventional ultrasound of 10 cases with lacrimal sac cyst showed non-uniform, hypoechogenic masses, or cystic solid mixed masses with clear edge but no blood flow signal; the CEUS displayed peripheral circular enhancement and no enhancement inside. Results of conventional ultrasound of 3 cases with adenocarcinoma of lacrimal sac showed hypoechogenic solid masses with unclear edge, irregular form, non-uniform echo
\end{abstract}

Correspondence to: Dr Wen Xiong or Dr Qin Chen, Department of Ultrasound Diagnosis, Sichuan Academy of Medical Sciences and Sichuan Provincial People's Hospital, 32 West First Ring Road, Chengdu, Sichuan 610072, P.R. China

E-mail:vud441@163.com

E-mail: chenqin630528@sina.com

Key words: lacrimal apparatus, tumor, color Doppler ultrasound, contrast-enhanced ultrasound inside, and abundant blood flow signals in lacrimal sac; CEUS displayed high, fast-developed fast-extinct and overall uniform enhancement; after enhancement, masses with irregular shapes were obviously larger than that in two-dimensional ultrasound. CEUS shows the microcirculation of tumors and surrounding tissues. Combination of two-dimensional and color Doppler ultrasound can improve the preoperative qualitative diagnosis of tumors and provide references for the selection of operation methods and determination of tumor resection scope.

\section{Introduction}

Lacrimal apparatus includes lacrimal gland and passage, and lacrimal passage includes lacrimal punctum, ductule, sac and naso-lacrymal duct. Lacrimal gland exerts the secretion function, while lacrimal passage exerts the excretory function. Lacrimal gland mass is rare, and lacrimal gland mass in palpebral part is even rarer than that in orbital part; but incidence of tumors is the highest in lacrimal gland diseases. Inflammatory lesions are common in lacrimal passage diseases, but malignant tumors are rare. Color Doppler ultrasound applied in ophthalmic diseases can not only clearly show the two-dimensional structures of eyeballs and surrounding orbital wall tissues, but also display the normal and abnormal blood flow conditions. Contrast-enhanced ultrasound (CEUS) is a kind of imaging technique that the ultrasonic contrast agent containing microbubbles is intravenously injected to enhance the back-scattered blood flow signal in human body, which can observe the microvascular perfusion in tissues in a real-time and dynamic way, thereby providing more ultrasound diagnostic information. At present, CEUS has been widely applied in the diagnosis of liver tumors. However, the application of CEUS in superficial organ tumors, especially eye tumors, is still in the exploratory stage. Moreover, reports on CEUS applied in lacrimal apparatus tumors are rare. This study investigated the application values of two-dimensional, color Doppler ultrasound and CEUS in the diagnosis of lacrimal apparatus tumors.

\section{Patients and methods}

Objects of study. A total of 48 patients pathologically and clinically diagnosed with lacrimal apparatus tumors were 
treated in China Meitan General Hospital (Beijing, China) from July 2016 to December 2017 underwent two-dimensional, color Doppler ultrasound and CEUS examinations before operation, among which there were 18 males and 30 females with an average age of 38 years. This study was approved by the Ethics Committee of China Meitan General Hospital (Beijing, China) and written informed consents were signed by the patients or their guardians.

Instruments and methods. MyLab 90 color Doppler ultrasound diagnostic apparatus (Esaote SpA, Genoa, Italy) was used, and electronic linear probe LA523 with a frequency of 4-13 MHz was used in two-dimensional and color Doppler ultrasound. Real-time contrast-tuned imaging (CnTI) technique was equipped in the apparatus, and probe LA522 (3-9 MHz) was used in CEUS. SonoVue (SF6) manufactured by Bracco (Milan, Italy) was used as ultrasonic contrast agent. The average diameter of microbubbles was $2.5 \mu \mathrm{m}$, and the contrast agent $(59 \mu \mathrm{g})$ was used to make suspension (SF6 concentration, $5 \mathrm{mg} / \mathrm{ml}$ ) with $5 \mathrm{ml}$ normal saline. After intravenous bolus injection of contrast agent, $5 \mathrm{ml}$ normal saline was injected, and patients were continuously observed for 3-5 min. Dynamic images were saved for later analysis. Before contrast examination, the patients signed an informed consent.

Under a supine position, patients closed both eyes gently, and eyelids were coated with disinfectant coupling agent, followed by axial and non-axial scanning to observe site, shape, edge, size and echo of the mass, and color Doppler ultrasound was used to observe the blood supply and other features of the mass. Then the optimal section of the mass (the mass and some normal choroid were shown in one section as far as possible) was selected, and the contrast agent was injected under the contrast mode; at the same time, the built-in timer of ultrasonic apparatus was started, and the mass and surrounding tissues were observed in real-time for $\sim 3-5 \mathrm{~min}$; the whole process was videotaped.

According to CEUS features of 48 cases of masses, enhancement degrees of masses were divided into three types: i) Uniform enhancement: uniform filling of contrast agent in the mass; ii) non-uniform enhancement: Non-uniform filling of contrast agent in the mass; non-filling area could be seen inside; and iii) No enhancement: No filling of contrast agent in the mass. There were three types of enhancement modes: i) Overall enhancement: Overall enhancement of filling of contrast agent in the mass; ii) centripetal enhancement: gradual enhancement of filling of contrast agent in the mass from periphery area to inner area; and iii) circular enhancement: Circular enhancement of contrast agent only around the mass. With the filling and extinction time of contrast agent in normal retina and choroid near the mass as control, the filling and extinction time of contrast agent in the mass was observed: i) Rapid enhancement of the mass, named fast-developed: The filling time of contrast agent in the mass is the same or shorther than that in surrounding normal retina and choroid; ii) rapid extinction of the mass, named fast-extinct: The extinction time of contrast agent in the mass is shorther than that in surrounding normal retina and choroid; and iii) slow extinction of the mass, named slow-extinct: The extinction time of contrast agent in the mass is longer or the same as that in surrounding normal retina and choroid (1).
Table I. Analysis of two-dimensional ultrasound and CEUS imaging of 29 cases of lacrimal gland pleomorphic adenoma (n, \%) (Fig. 1).

\begin{tabular}{lc}
\hline Types & Cases \\
\hline Male & $9(31.03)$ \\
Female & $20(68.97)$ \\
Age (years) & \\
$<30$ & $20(68.97)$ \\
$30-50$ & $3(10.34)$ \\
$>50$ & $22(75.86)$ \\
Clear edge & $28(96.55)$ \\
Unclear edge & $1(3.45)$ \\
Internal echo dense and uniform & $20(68.97)$ \\
Inhomogeneous calcification & $6(20.69)$ \\
Echoic sheets & $3(10.34)$ \\
Internal dotted blood flow signal & $29(100)$ \\
$\begin{array}{l}\text { Contrast-enhanced overall uniformity, } \\
\text { fast forward and slow retreat }\end{array}$ & $20(68.97)$ \\
High homogeneity of centripetality, & \\
fast forward and slow retreat & $6(20.69)$ \\
High inhomogeneity of centripetality, & \\
fast forward and slow retreat & $3(10.34)$
\end{tabular}

CEUS, contrast-enhanced ultrasound.

Table II. Analysis of two-dimensional ultrasound and CEUS for 10 cases of lacrimal cysts (n, \%) (Fig. 3A and B).

\begin{tabular}{lc}
\hline Types & Cases \\
\hline Clear edge & $10(100)$ \\
Internal dot-strip strong echo & $7(70)$ \\
Capsule mixed echo & $3(30)$ \\
Rear enhancement & $10(100)$ \\
Non-internal blood flow signal & $10(100)$ \\
Peripheral dotted blood flow signal & $3(30)$ \\
No enhancement in the interior, & $10(100)$ \\
mild enhancement in the peripheral & \\
capsule walls, and circular enhancement & \\
in the enhancement &
\end{tabular}

CEUS, contrast-enhanced ultrasound.

\section{Results}

The 48 pathologically diagnosed masses were 29 cases with pleomorphic adenoma of lacrimal gland, 6 cases with adenoid cystic carcinoma of lacrimal gland, 10 cases with lacrimal sac cyst, and 3 cases with adenocarcinoma of lacrimal sac (Table I).

Results of conventional ultrasound of 29 cases with pleomorphic adenoma of lacrimal gland showed 
Table III. CEUS features of 48 cases of lacrimal apparatus tumors.

\begin{tabular}{lrl}
\hline Pathological diagnosis & No. & \multicolumn{1}{c}{ Enhancement mode } \\
\hline Pleomorphic adenoma of lacrimal gland & 20 & $\begin{array}{l}\text { High, fast-developed slow-extinct and overall uniform enhancement } \\
\text { High, fast-developed slow-extinct, centripetal, uniform } \\
\text { or non-uniform enhancement } \\
\text { High, fast-developed fast-extinct, overall uniform enhancement }\end{array}$ \\
Adenoid cystic carcinoma of lacrimal gland & 9 & $\begin{array}{l}\text { Circular enhancement, no enhancement inside } \\
\text { Lacrimal sac cyst }\end{array}$ \\
Adenocarcinoma of lacrimal sac & 10 & High, fast-developed fast-extinct, overall uniform enhancement \\
\hline
\end{tabular}

CEUS, contrast-enhanced ultrasound.
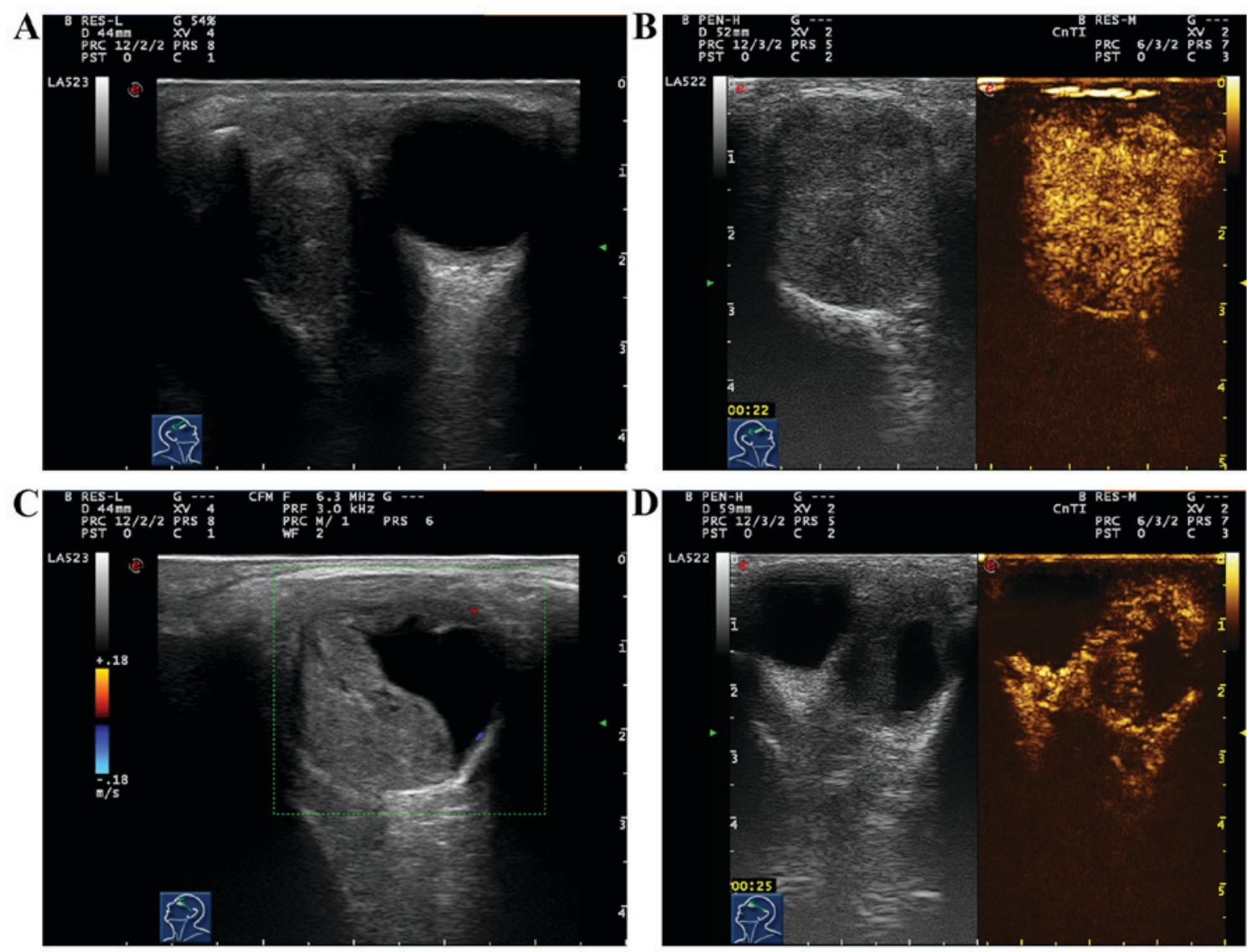

Figure 1. (A) Two-dimensional ultrasound image of pleomorphic adenoma of lacrimal gland. Solid hypoechoic mass with clear boundary, regular shape, and homogeneous internal echo. (B) CEUS image of pleomorphic adenoma of lacrimal gland at the peak (22 sec). (C) Pleomorphic adenoma of lacrimal gland Solid hypoechoic mass with clear boundary, regular shape, and homogeneous interior with flaky hypoechoic area. CDFI: spot blood flow signal can be seen around the mass. (D) Pleomorphic adenoma of lacrimal gland $(25 \mathrm{sec})$. CEUS, contrast-enhanced ultrasound.

moderate-hypoechogenic solid masses in lacrimal gland. Twenty-eight cases $(28 / 29,96.55 \%)$ had clear mass edge, while 1 case $(1 / 29,3.45 \%)$ had unclear mass edge and irregular shape. Twenty cases $(20 / 29,68.97 \%)$ had dense and uniform echo inside, 6 cases $(6 / 29,20.69 \%)$ had non-uniform echo inside accompanied by scattered calcification, and 3 cases $(3 / 29,10.34 \%)$ had echoic sheets. Results of color Doppler ultrasound of blood flow displayed that 29 cases $(29 / 29,100 \%)$ had dotted blood flow signal inside. After two-dimensional and color Doppler ultrasound, CEUS was immediately performed for 29 cases with pleomorphic adenoma of lacrimal gland.
Results showed that 20 cases $(20 / 29,68.97 \%)$ had high and overall uniform enhancement in masses; after enhancement, mass edge was clearer in regular shape, and the contrast agent within the mass became extinct slowly; the enhancement mode was 'high, fast-developed slow-extinct and overall uniform enhancement'. Nine cases $(9 / 29,31.03 \%)$ showed gradual enhancement of masses from the periphery to the center, and 6 cases showed uniform enhancement, and 3 cases showed non-uniform enhancement; there was non-enhancement area inside, and after enhancement, the mass edge was clear in regular shape, and retreat of contrast agent within the 

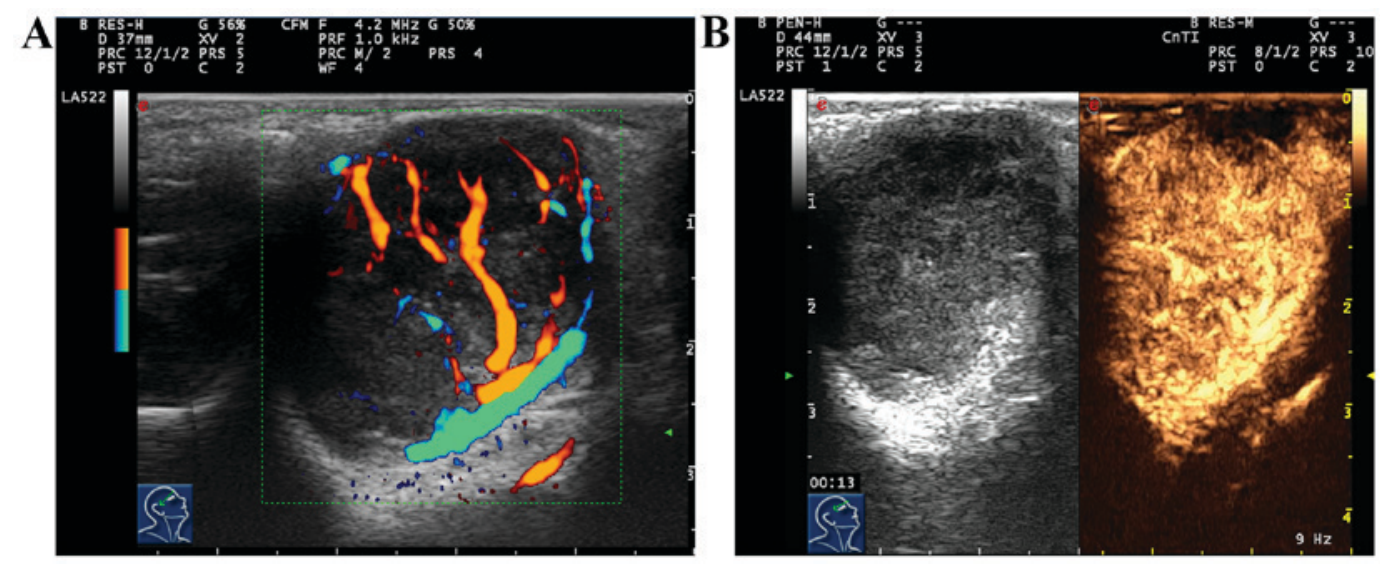

Figure 2. (A) Adenoid cystic carcinoma of lacrimal gland: Multiple thick vasa vasorum in lacrimal gland mass. (B) Adenoid cystic carcinoma of lacrimal gland: high overall uniform enhancement in CEUS at the peak $(13 \mathrm{sec})$. CEUS, contrast-enhanced ultrasound.
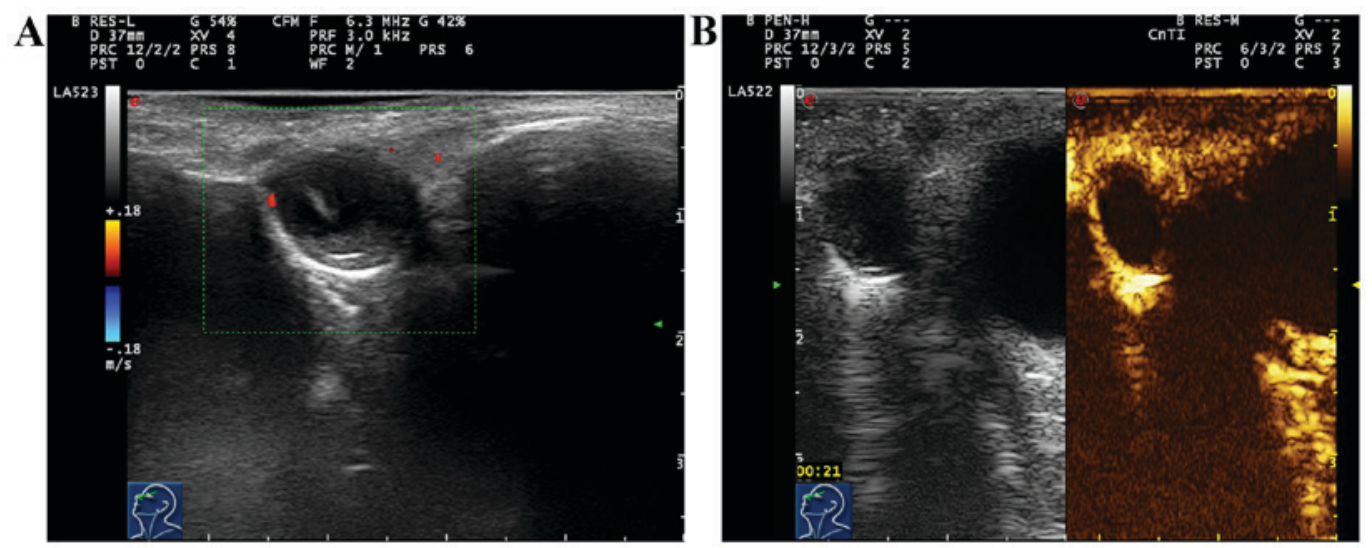

Figure 3. (A) Color flow image of lacrimal sac cyst. Punctate blood flow signal is seen around the tumor. (B) CEUS image of lacrimal sac cyst at the peak $(21 \mathrm{sec})$. CEUS, contrast-enhanced ultrasound.

mass became slowly extinct; the enhancement mode was high, fast-developed slow-extinct, centripetal, uniform or non-uniform enhancement' (Fig. 1).

Results of conventional ultrasound of 6 cases with adenoid cystic carcinoma of lacrimal gland showed hypoechogenic solid masses with unclear edge, irregular form and non-uniform echo inside the mass. Color Doppler ultrasound of blood flow displayed abundant blood flow signals inside the mass, and 6 cases had thick and tortuous vasa vasorum in the mass (Fig. 2A). After two-dimensional and color Doppler ultrasound, CEUS was immediately performed for 6 cases with adenoid cystic carcinoma of lacrimal gland. Results showed that 6 cases $(6 / 6,100 \%)$ had stong and overall uniform enhancement in masses; in the early stage of enhancement, thick vasa vasorum could be seen around and inside the mass; after enhancement, the mass was larger than that in two-dimensional ultrasound, and retreat of contrast agent within the mass became extinct fast; the enhancement mode was 'high, fast-developed fast-extinct, overall uniform enhancement' (Fig. 2B; Table II).

Ten cases with lacrimal sac cyst had clear mass edge, among which 7 cases $(7 / 10,70 \%)$ showed moderate non-uniform hypoechogenic and dot-strip strong echo inside, and 3 cases $(3 / 10,30 \%)$ showed cystic solid mixed echo and wavy shape at the junction of mass and bone wall. Ten cases $(10 / 10,100 \%)$ had enhancement behind the mass. Color Doppler ultrasound of blood flow displayed that 10 cases $(10 / 10,100 \%)$ had no blood flow signal inside the mass, and 3 cases $(3 / 10,30 \%)$ had dotted blood flow signal around the mass. After two-dimensional and color Doppler ultrasound, CEUS was immediately performed for 10 cases with lacrimal sac cyst. Results showed that there was no enhancement in the mass, mild enhancement could be seen around the mass, and the enhancement mode was 'circular enhancement' (Fig. 3A and B; Table III).

Results of conventional ultrasound of 3 cases with adenocarcinoma of lacrimal sac showed hypoechogenic solid masses, and superficial subcutaneous masses with unclear edge, irregular form, non-uniform echo inside, scattered dotted strong echo and incomplete periostea on the edge. Color Doppler ultrasound of blood flow displayed abundant blood flow signals inside the mass. After two-dimensional and color Doppler ultrasound, CEUS was immediately performed for 3 cases with adenocarcinoma of lacrimal sac. Results showed that the appearance of enhancement in mass was obviously earlier than that in surrounding normal retina and choroid, displaying overall uniform high enhancement; after enhancement, mass was larger than that observed in two-dimensional ultrasound, and retreat of contrast agent within the mass became extinct fast; the enhancement mode was 'high, fast-developed fast-extinct, overall uniform enhancement' (Fig. 4A and B). 

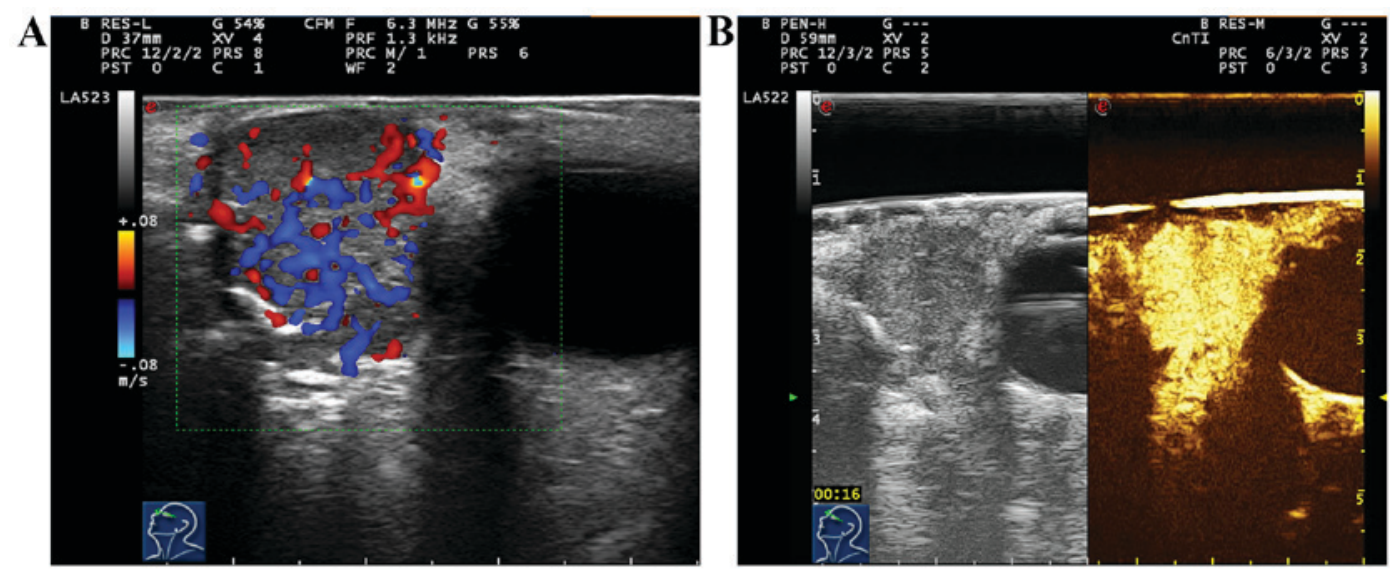

Figure 4. (A) Color flow image of adenocarcinoma of lacrimal sac. There are abundant irregular blood flow signals inside and around the tumor. (B) Adenocarcinoma of lacrimal sac: High overall uniform enhancement in CEUS at the peak (16 sec) (with water sac). CEUS, contrast-enhanced ultrasound.

\section{Discussion}

Unrestricted growth of malignant tumors depends on internal neovascularization. Ultrasound contrast agent is a kind of intravascular contrast agent, which can display well the distribution of microcirculation inside the tumor and reflect the abundance of neovascularization inside the tumor. Thus, CEUS was used in the evaluation of blood perfusion inside the tumor. Moreover, the feature of CEUS also compensates the shortcomings of traditional two-dimensional and color Doppler ultrasound. Color Doppler flow imaging is sometimes affected by blood flow direction within the lesion and the poor sensitivity of instrument to low-speed blood flow, and alse-negative blood flow is also observed in some lesions. Therefore, no blood flow signal in color Doppler ultrasound does not indicate no blood supply lesion in the mass (2). Application of CEUS imaging technique makes ultrasound diagnostic instrument display clearer microcirculation within lesion, providing a new method for the diagnosis of ophthalmic space-occupying lesions $(3,4)$.

Yang et al applied ultrasound contrast agent to the human eye after relevant animal experiments, and confirmed that its application in the eye did not cause local and systemic side-effects, and also had no significant impact on retinal function and structure (5).

Lacrimal apparatus tumor includes lacrimal gland and passage tumor. The most common lacrimal gland tumor is the primary epithelial lacrimal gland tumor, among which pleomorphic adenoma of lacrimal gland is common in benign tumors, and adenoid cystic carcinoma of lacrimal gland is common in malignant tumors (6).

Pleomorphic adenoma of lacrimal gland is the most common lacrimal gland epithelial tumor, accounting for $\sim 50 \%$. It is a kind of benign tumor composed of epithelial and interstitial components. In the past, pleomorphic adenoma of lacrimal gland was called benign mixed tumor. It occurs frequently in single eye in adults and histopathological components mainly include epithelial cells or interstitial components. Besides, it is composed of a large number of tubular structures and cell nests in different shapes formed by differentiated epithelial cells, with scattered transparent myxoid and cartilage-like structure, diversified forms and arrangements of tumor cells (7). Ultrasound shows the round or oval solid mass above the orbit, and the majority of masses have clear edge and dense and uniform echo inside, but a small number of masses have unclear edge and non-uniform echo inside with scattered calcification; the mass is not compressed with a small number of blood flow signals. CEUS shows rapid filling of contrast agent within the mass, most of which show strong overall uniform enhancement, and a few of which show concentric uniform or non-uniform enhancement; after enhancement reaches the peak, retreat of contrast agent becomes slowly extinct. In this study, it was found that the mass containing more epithelial and mucinous tissues showed high and uniform enhancement after CEUS, while the mass containing more cartilage-like structures showed high and non-uniform enhancement after CEUS, and flaky non-enhancement area could be seen within the mass, and the proportion of cartilage-like structure was directly proportional to the range of non-enhancement area within the mass. Differential diagnosis of the disease is as follows: i) Inflammatory pseudotumor of lacrimal gland: It often occurs in both eyes, showing eyelid congestion and edema; hormone therapy is effective but recurrence rate is high. Ultrasound examination shows lacrimal gland enlargement in a flat or amygdaloidal shape, and it can extend forward or towards the orbital apex, often accompanied by adjacent extraocular muscle thickening. CEUS shows that after enhancement, the mass edge is unclear, the mass is larger than that observed in two-dimensional ultrasound, and the lesion generally has no enhancement area; ii) lymphoma of lacrimal gland: Orbital lymphoma can often occur in the lacrimal gland. Ultrasound examination shows the low and non-uniform echo in the lesion, and scattered cord-like strong echo. Doppler detection shows that the mass has abundant blood signal in a dendritic shape. Spectral Doppler shows that the high-speed high-resistance arterial spectrum is an imaging feature of orbital lymphoma, which is of significance in the early diagnosis and timely treatment of lymphoma. Moreover, CEUS shows that most tumors develop and disappear quickly, and show high uniform enhancement; after enhancement, mass edge is unclear, and the mass is larger than that observed in two-dimensional ultrasound, and the posterior edge of lesion often shows an 'inverted-triangle' shape $(1,8)$.

Adenoid cystic carcinoma of lacrimal gland is the most common malignant epithelial tumor of lacrimal gland; the mass 
is solid with no capsule and has a high degree of malignancy. It often invades surrounding tissues, and relapse and metastasis occur easily. This disease is considered as the most biologically destructive and unpredictable tumor in the head and neck (8). Ultrasound shows solid hypoechogenic mass in a spindle shape with unclear edge and irregular form without capsule surrounding the mass; internal echo is not uniform, and slightly attenuated in the rear; abundant blood flow signals and multiple thick vasa vasorum can be detected in the mass $(9,10)$. In CEUS, the contrast agent in the mass is rapidly filled, showing high overall uniform enhancement; after enhancement, the mass is enlarged and retreat of contrast agent becomes extinct quickly after enhancement reaches the peak, showing fast-developed fast-extinct signs, which is different from pleomorphic adenoma and tuberculosis of lacrimal gland (fast-developed slow-extinct). However, the number of cases with such a sign is small, so it cannot be used as a criterion to distinguish benign from malignant lacrimal gland masses, but may provide references for the diagnosis of malignant tumors. Differential diagnosis of the disease is the same as that of pleomorphic adenoma of lacrimal gland; but it is hard to distinguish it from inflammatory lesions from two-dimensional ultrasound and CEUS, so the clinical manifestations, signs and past medical history should be combined for comprehensive analysis.

Stupp et al (11) performed ultrasound examination for 17 patients with lacrimal sac mass, and the results showed that ultrasound could display the lacrimal sac mass well. The disease should be distinguished from frontal and ethmoidal sinus cysts, which are easily misdiagnosed as ophthalmic diseases.

Lacrimal passage tumor is rare, and the malignant lacrimal passage tumor is even rarer, and the main pathological types are squamous and transitional cell carcinoma, followed by adenocarcinoma. Typical clinical manifestations of lacrimal passage tumor are masses in inner canthus or lacrimal sac, and it only displays the overflow of tears and unblocked lacrimal passage in the early stage. Clinical diagnosis of this disease is difficult. With the progression of disease, pain, overflow of lacrimal punctum and bloody mucus, lacrimal sac area bulges will occur; in the late stage, it may lead to epistaxis, protopsis and other complications. Ultrasound of malignant lacrimal passage tumor shows no specificity, and only shows the solid non-uniform mass in lacrimal sac. In addition, different pathological types of tumors show different strengths of blood flow signal; generally, blood flow signal of squamous cell carcinoma is low, while poorly-differentiated adenocarcinoma shows more abundant signals. The sample size in this study was small, and its sensitivity, specificity and accuracy were not discussed, so the results could not be used as criteria to distinguish benign from malignant lacrimal apparatus tumors. Therefore, future studies with larger number of samples are needed to further confirm the conclusion.

In conclusion, traditional two-dimensional and color Doppler ultrasound may be combined with CEUS in the determination of mass size, edge and shape. This technology may facilitate the identification of tumor scope and improve the diagnosis.

\section{Acknowledgements}

Not applicable.

\section{Funding}

No funding was received.

\section{Availability of data and materials}

The datasets used and/or analyzed during the present study are available from the corresponding author on reasonable request.

\section{Authors' contributions}

YXL wrote the manuscript and treated the patients, YL and JMX were responsible for the ultrasound diagnosis. QC and WX helped with the statistical analysis. All authors read and approved the final manuscript.

\section{Ethics approval and consent to participate}

The study was approved by the Ethics Committee of China Meitan General Hospital (Beijing, China). Signed informed consents were obtained from the patients or the guardians.

\section{Patient consent for publication}

Not applicable.

\section{Competing interests}

The authors declare that they have no competing interests.

\section{References}

1. Chen Q, Zhou G, Xiong W, Zhou Q and Yue LX: Ultrasound and CEUS features of orbital and intraocular mass. Shengxue Jishu 30: 466-470, 2011 (In Chinese).

2. Yang Q and Wei WB: CEUS and its clinical application in ophthalmology. Int Rev Ophth 31: 145-148, 2007.

3. Tu Y, Jakobiec FA, Leung K and Freitag SK: Distinguishing benign from malignant circumscribed orbital tumors in children. Semin Ophthalmol 33: 116-125, 2018.

4. Li HD and Fu P: Application of CEUS in ophthalmology. Int Rev Ophth 34: 94-97, 2010.

5. Yang Q, Wei WB, Yang WL, Shi XH, Zhou D and Liu Y: Experimental research on effects of SonoVue real-time CEUS on choroidal retina structure and function. Ophthalmology 17: 193-197, 2008

6. Alkatan HM, Al-Harkan DH, Al-Mutlaq M, Maktabi A and Elkhamary SM: Epithelial lacrimal gland tumors: A comprehensive clinicopathologic review of 26 lesions with radiologic correlation. Saudi J Ophthalmol 28: 49-57, 2014.

7. Tailor TD, Gupta D, Dalley RW, Keene CD and Anzai Y: Orbital neoplasms in adults: Clinical, radiologic, and pathologic review. Radiographics 33: 1739-1758, 2013.

8. Bischof M, Karagiozidis M, Krempien R, Treiber M, Neuhof D, Debus J and Zierhut D: Radiotherapy for orbital lymphoma: outcome and late effects. Strahlenther Onkol 183: 17-22, 2007.

9. Feng LL, Xian JF, Yan F, Fu L and Zhou HY: Value of DCE-MRI and DWI in the differential diagnosis of inflammatory pseudotumor and lymphoma in the lacrimal gland. Zhonghua Yi Xue Za Zhi 97: 487-491, 2017 (In Chinese).

10. Yan L, He G, Zhou X, Zheng Y, Zhu Y, Yang J, Zhang M and Zhou Y: Contrast-enhanced ultrasound in the diagnosis of orbital space-occupying lesions. Clin Radiol 72: 798.e1-798.e6, 2017.

11. Stupp T, Pavlidis M, Busse H and Thanos S: Presurgical and postsurgical ultrasound assessment of lacrimal drainage dysfunction. Am J Ophthalmol 138: 764-771, 2004. 